\title{
MARSIPAN-based Early Warning Signs System: A full Audit cycle in the Inpatient Eating Disorders Ward
}

Konstantinos Ioannidis ${ }^{\mathrm{a}, \mathrm{b}}$, Gabriella Lewis ${ }^{\mathrm{a}}$, Jane Waterston ${ }^{\mathrm{b}}$, Carol Connolly $\mathrm{y}^{\mathrm{b}}$, Felix Clay ${ }^{\mathrm{b}}$, Chloe Copping ${ }^{\mathrm{b}}$, Alex Gomez-Mendes ${ }^{\mathrm{b}}$, Charlie Middleton ${ }^{\mathrm{b}}$, Jaco Serfontein ${ }^{\mathrm{b}}$

a University of Cambridge, Department of Psychiatry

b Cambridge and Peterborough NHS Foundation Trust

Correspondence: Dr Konstantinos Ioannidis, S3 Eating Disorders, Addenbrookes Hospital, Hills Road, Cambridge, UK, CBQ Q20; ioannik@doctors.org.uk 


\section{Abstract}

Aims and method: We aimed to evaluate the MARSIPAN-guidance-adapted Early Warning Sign system (MARSI-MEWS) in respect to clinical governance standards for accuracy and completion. We performed a full audit cycle; between audit rounds, we addressed intrinsic information design problems related to the form in operation and provided guidance to staff on how to use this specialized tool.

Result: Overall completeness of recording and scoring of MARSI-MEWS improved from $82.0 \%$ to 99.5\% $\left(\chi^{2}, \mathrm{p}<0.001\right)$. The accuracy of MARSI-MEWS scoring improved from $80 \%$ to $93 \%\left(\chi^{2}\right.$, $\mathrm{p}<0.001)$. False recording of diastolic blood pressure dropped from $98 \%$ to $0 \%\left(\chi^{2}, \mathrm{p}<0.001\right)$.

Clinical implication: This result suggests that the MARSI MEWS track-and-trigger system is suitable for use in inpatient eating disorders ward in regard to its completeness and accuracy standards. Specific training for staff might be essential in order to complete the tool effectively, and the validity of the tool should be tested in future work.

Keywords: anorexia, early warning signs, inpatient, deterioration 


\section{Introduction}

Anorexia nervosa (AN) has the highest morbidity and mortality amongst mental illnesses (1). Severe anorexia nervosa often treated in the inpatient setting to prevent medical deterioration. AN has an estimated standardized mortality ratio of $\sim 5.8$ (1) and a high degree of medical complications, including sudden deaths by cardiovascular collapse (2). Therefore, when treating AN patients in the inpatient settings, it is all important to measure physiological elements of risk that drive the main morbidity and mortality risk factors. Therefore, the routine measurement of physiological parameters is essential in eating disorders inpatient facilities, despite the fact that the importance of physical observations in the inpatient settings is often underestimated (3).

Track-and-trigger systems have been developed to ascertain whether there is potential or established critical illness, so that clinically deteriorating cases are early identified and appropriately skilled clinical leads are ensured to follow through the management of such cases $(4,5)$. National track-and-trigger systems for monitoring clinical and were developed since the turn of the century (6) and established UK-wide as the National Early Warning Signs system (NEWS) (7). It is furthermore recommended that track and trigger systems should be reviewed regularly to optimise sensitivity and specificity. Furthermore, the NEWS Development and Implementation group (NEWSDIG) specifically highlighted that the NEWS could not provide a sensitive identification of cases for populations with chronically altered physiology (8); this is a characteristic of the AN population. Furthermore, the NEWSDIG recommended that protocols should specifically be tailored to accommodate for the markedly different physiological responses. AN patients have been described to demonstrate lower systolic blood pressure (BP), slower heart rates (HR) and lower core temperatures (T) when compared to age-and-gendermatched healthy controls $(9,10)$. Furthermore, AN adults can present with abnormalities in lower left ventricular mass and filling indicating a dysfunction of the contractility and pumping activity of the heart $(11,12)$. Therefore, the development of specialized track-and-trigger early warning signs system tailored to the AN populations is an essential task at hand. In view of the requirement to develop a tailored specialized system, the PRIORY group (13), developed the 'MARSI MEWS' (MARSIPAN-guidance-based Modified Early Warning Sign System (14)), based on the 'Guide to the medical risk assessment for eating disorders' (15) and the Physical Risk in Eating Disorders Index (PREDIX) (16), as well consultation with MARSIPAN physicians.

The MARSI MEWS were consecutively adopted by S3 Eating Disorders Service of Cambridge and Peterborough NHS Foundation Trust as best practice and replaced the NEWS as a track-andtrigger system in the service. While CPFT adopted the MARSI MEWS track-and-trigger system, there still remained the question of whether the tool is valid, and whether it is appropriate for 
use in an eating disorder inpatient setting. Most importantly, when the MARSI MEWS were originally published, McCluskey et al. reported significant issues with the completeness of the tool (only 50\% total completeness) and accuracy of MEWS scoring (only 36\%)(13). This raises concerns about whether the tool is appropriate for use in an inpatient eating disorders setting and the degrees of completeness and accuracy need to be established.

By setting out to perform a full audit cycle, we aimed to assess if current CPFT practice is keeping in line with national standards for clinical safety. As secondary goal, we wanted to ascertain if there are interventions that would allow us to further improve the completeness and accuracy of the tool. A tertiary goal was to ascertain summary statistics (sensitivity, positive predictive value) from the use of Sepsis indicator.

\section{Methods}

\section{Audit rationale and standards}

The audit was undertaken in line with the general ideas outlined by the National Quality Board: Review of early warning systems in the NHS Acute and community services and recommendations provided by the Quality Network in Eating Disorders (17). The standards used in this audit were the National Early Warning Score (NEWS)(8), the MARSIPAN: Management of Really Sick Patients with Anorexia Nervosa 2nd edition guidance (14) and the Cambridge and Peterborough NHS Foundation Trust (CPFT) guidance on use of the MARSIPAN Adapted Early warning score (MARSI MEWS) for the S3 Inpatient Adult Eating Disorders Unit.

\section{Setting and participants}

For the first round of the audit, data collection included all inpatients of S3, Inpatient Eating Disorder Ward, Addenbrookes Hospital, which were hospitalized during the month of September 2016. This included 13 inpatients and one day-patient who had physical observations taken as part of their clinical care, and had MEWS scores calculated and acted upon accordingly. During this first round we collected 299 observations in total. In round 2 (re-audit) we gathered measurements from all inpatients of S3, Inpatient Eating Disorder Ward, Addenbrookes Hospital, which were hospitalized during the month of May 2017. During this second round we collected 364 observations in total from 15 inpatients. 


\section{Measures}

We collected anonymised data relating to patient identifiers i.e. audit ID, RIO number, also, physiological parameters i.e. Pulse Rate/Heart rate (HR), Blood pressure (BP) systolic, Blood glucose measurement (BM), Respiratory Rate (RR), oxygen saturation (SO2\%), Temperature (T), alertness (AVPU) and postural BP drop that enabled the MARSI MEWS scoring. We also noted whether diastolic blood pressure was recorded or not. The physical parameter variables were directly extracted from the MARSI MEWS forms. We also collected White blood cell count (WBC) from the RIO electronic records.

\section{Ethics}

The audit was approved by the local NHS service (Cambridge and Peterborough NHS Foundation Trust) and registered as a clinical audit by the Quality Assurance and Clinical Effectiveness Team.

\section{Analysis}

Anonymized data was initially collected in a secure server excel file and entered into Statistical Analysis software R version 3.1.2. The paper recorded physical observations scores were used to calculate a correct (post-hoc) MARSI MEWS score and assess accuracy of scoring. The scoring system for Round 1 is available for online download from McCluskey et al. (13). The amended form with information design changes is presented in the supplemental material MARSI-MEWS 2.0 form). We dealt with missing values by scoring them as null values i.e. not adding to the total MEWS score. Recorded MEWS and post-hoc calculated MEWS were compared by simple case-bycase comparison and classified as binary i.e. correctly and not-correctly classified. The noncorrectly classified were further identified as 'wrongly scored' i.e. MEWS score being incorrectly calculated and 'not scored' i.e. MEWS score missing. The Sepsis indicator score was also calculated by the different physiological parameters according to the sepsis indicator scoring system and each observation was characterized as 'non-case' (less than two positive indicators of sepsis) or 'case' (two or more indicators of sepsis). Sepsis indicators included: 1) Respiratory rate above 20/min, 2) Heart Rate above $>90,3$ ) Temperature above 38.3C or below 36C, 4) WBC above $12^{*} 10^{\wedge} 9 / \mathrm{l}$ or below $\left.4^{*} 10^{\wedge} 9 / \mathrm{l}, 5\right)$ Non alert alertness score, and 6) Blood glucose above $7.7 \mathrm{mmol} / \mathrm{l}$.

\section{Results}

The mean score and range from each parameter that was collected is presented in Table 1. 


\section{[INSERT TABLE 1 ABOUT HERE]}

\section{First round results}

We compared the reported MARSI MEWS score with the ad-hoc true score and reported a classification accuracy of $80 \%(n=299)$, with 9\% $(n=27)$ wrong MARSI MEWS scores and 11\% $(\mathrm{n}=32)$ missing MEWS scores. We identified that a significant percentage of physical observations recordings included diastolic blood pressure (98\%) ( $n=293)$, which is not used in MEWS score calculation, whereas an equally significant number of observations did not included postural drop of blood pressure (96\%) ( $n=287)$, which should be used in MEWS calculation. This implies that the vast majority of cases lacked postural drop data in the MEWS calculation, and there is a possibility that the recording of diastolic blood pressure might have influenced errors in calculation due to the possibility staff scoring this observation according to the blood pressure scoring indicators. Indeed, on inspection of the MARSI MEWS forms we identified instances for which staff may have erred because of that (i.e. scored '3' points for diastolic BP).

\section{First round recommendations}

The first round of the audit resulted in specific recommendations: 1) Staff performing the physical observations should be mindful of performing physical observations in completeness and scoring the MARSI MEWS; 2) Staff should be mindful that diastolic pressure recording is not necessary to score the MEWS and this may contribute to scoring errors; 3 ) The usefulness and appropriateness of the Sepsis indicator should be re-considered for use by non-medical ward staff; 4) The accuracy and completeness of MARSI MEWS is subject to improvement; 5) The MEWS form can be redesigned to facilitate accurate scoring. The implementation of changes and training of staff to use the amended form was scheduled from Feb-May 2017; the re-audit was planned for and performed in May 2017.

\section{Second Round (re-audit) Results}

In round 2 we compared the calculated MEWS score with the true (post-hoc) score calculated by the paper-recorded physical observations and this gave a classification accuracy of $92.8 \%$ $(\mathrm{n}=338)$ with $6.0 \%(\mathrm{n}=22)$ wrong MEWS scores and 1.1\% $(\mathrm{n}=4)$ missing MEWS scores. Physical observations recordings which included diastolic blood pressure dropped from $98 \%(n=293)$ to $0 \%(n=364)$. Postural drop of blood pressure recording improved to $99.7 \%(n=363)$. There were improvements in other areas as well for example recording of respiratory rate (RR) increased from $80 \%$ to $99 \%$ [ $\left.\chi^{2}, \mathrm{p}<0.001\right]$, oxygen saturation from $96 \%$ to $98.6 \%\left[\chi^{2}, \mathrm{p}<0.5, \mathrm{p}\right.$ corrected $>$ $.05]$, heart rate from $96 \%$ to $99.7 \%\left[\chi^{2}, \mathrm{p}<0.001\right.$, p corrected $\left.<.01\right]$, systolic BP recording improved from 97.3 to $100 \%\left[\chi^{2}, \mathrm{p}<0.01\right.$, p corrected $\left.<.05\right]$, temperature from $96 \%$ to $100 \%\left[\chi^{2}\right.$, $\mathrm{p}<0.001$, p corrected <.01], AVPU from 97.7 to $100 \%\left[\chi^{2}, \mathrm{p}<0.05\right.$, p corrected $\left.>.05\right]$. These 
changes were expected to improve accuracy of scoring which indeed this improved from $80 \%$ to $93 \%\left[\chi^{2}, \mathrm{p}<0.001, \mathrm{p}\right.$ corrected $\left.<.001\right]$.

\section{Sepsis indicator results}

In Round 1 , the Sepsis indicator identified 36 cases $(12 \%, \mathrm{n}=36)$ in our sample, whereas the true number of clinically defined sepsis cases was 0 (FPR $=12 \%, P P V=0 \%)$. If WBC count was not taken into account, the indicator would identify $3(1 \%, n=299)$ cases, implying that WBC might be the variable responsible for the high false positive rate. In round 2, the Sepsis indicator identified 0 cases in our sample that did not contain any true sepsis cases $(\mathrm{TNR}=100 \%, \mathrm{PPV}=$ $0 \%)$.

\section{[INSERT FIGURE 2 ABOUT HERE]}

\section{Discussion}

In our full audit cycle we identified correctable information design weaknesses in the MARSI MEWS form, which we addressed, and demonstrated a statistically significant improvement in completion of the instrument from $82.0 \%$ to $99.5 \%$ [ $\chi 2, \mathrm{p}<0.001]$, and MARSI MEWS scoring accuracy from $80 \%$ to $93 \%$ [ $\chi 2, \mathrm{p}<0.001]$. Therefore, we ascertained that the system can be adequately completed and scored accurately.

This is the first clinical effectiveness study to ever examine the use of an Early Warning sign system in a Specialised Eating Disorder inpatient treatment facility. Our results indicate that the MARSI MEWS system currently in operation on the ward can improve in terms of accuracy and completeness. This is suggestive that the MARSI MEWS adapted form (see online supplement), in conjunction with staff guidance could improve the usability of such specialized instrument; MARSI MEWS, if valid, can be a valuable tool for eating disorder units.

Our sepsis indicator results support the notion which is outlined in the MARSIPAN guidelines; AN populations present with atypical physiological and biochemical changes in sepsis; tools standardized in the general population can be liable to false positive errors, possibly due to the high frequency of leucopenia (Low WBC count) which is common among severely malnourished patients. AN patients often present with bone marrow suppression, which is a physiological adaptation to low body mass index and starvation, which means that they present with lower than usual WBC count, which can lead to a high false positive rates of sepsis identification. MARSIPAN recommends the inclusion of other physiological parameters indicative of risk of sepsis i.e. hypo-albuminaemia, or high C-Reactive Protein (CRP) to estimate risk of sepsis, 
although those are biochemical parameters monitored by trained medical staff. While our audit was not able to provide a comprehensive answer to the validity of the use of the sepsis indicator in this population group (due to lack of true sepsis cases in our sample), our results align with the notion that sepsis may present atypically in the AN population and specialized tools for early identification may be warranted and suggest that sepsis indicator should be used with caution, when used in isolation (as an instrument in its own merit) by non-medical staff.

According to the QED (1.8.6) standards for the physical health assessment, staff members should receive training consistent with their role, which includes taking physical observations from patients (17). To fulfil the MARSIPAN guidance requirement of being able to consistently monitor

BOX 1 - Key information design problems of MARSI MEWS identified and addressed

- The lack of clarity of score ranges in respect to the boxes e.g. each box needs to represent a range (e.g. RR of 12-20)

- Absence of glucose measurement scoring range

- Absence of noting area for taking record of standing blood pressure (influences the accuracy and completeness of postural drop calculation and scoring

- Information design suggestive of the requirement for diastolic blood pressure recording

glucose levels, postural drop and align the EWS with the AN-altered physiology, it can be argued that staff should receive specific guidance to allow them to use such specialized tool i.e. MARSI MEWS. While the measurement of those physiological and biological parameters is routine practice and embedded in staff training nationally, particular guidance for the use of MARSI MEWS should be provided. Due to the high affinity of the MARSI MEWS tool to the nationally utilized EWS system, our experience suggests that such guidance is not cumbersome and can dramatically help completion and accuracy of such instrument.

\section{Conclusions}

This is the first attempt for an audit of any service to explore the usefulness and practice of utilizing a MARSIPAN-based track-and-trigger system in the AN inpatient population. The audit showed that the tool is sufficiently completed and can be improved by the utilization of a newly designed form. Training of staff in using the new form is likely to be important to allow the new elements of the form to be utilized appropriately (e.g. noting the postural drop and glucose measurements). Providing guidance to staff and re-designing the form helped overall 
completeness of recording and accuracy of scoring of MARSI MEWS. Staff should be mindful that diastolic pressure recording is not necessary to score Early-warning-signs score.

\section{Limitations}

The audit did not address whether the MARSI MEWS system is valid for use; a larger scale review of the instrument is required to ascertain whether the MARSI MEWS is appropriate for use and how it compares to national standards e.g. the National Early warning signs system in terms of its capacity to identify deteriorating cases.

\section{Acknowledgements}

We are indebted to the inpatient team of S3 Eating Disorders, Addenbrookes Hospital, Cambridge and the Cambridge and Peterborough NHS Foundation Trust Quality Assurance and Clinical Effectiveness team, who supported this endeavour.

\section{Conflict of interest}

The authors have no conflict of interest to report 


\section{References}

1. Arcelus J, Mitchell AJ, Wales J, Nielsen S. Mortality Rates in Patients With Anorexia Nervosa and Other Eating Disorders. Arch Gen Psychiatry [Internet]. 2011 Jul 1 [cited 2019 Feb 18];68(7):724. Available from: http://www.ncbi.nlm.nih.gov/pubmed/21727255

2. Jáuregui-Garrido B, Jáuregui-Lobera I. Sudden death in eating disorders. Vasc Health Risk Manag [Internet]. Dove Press; 2012 [cited 2019 Feb 18];8:91-8. Available from: http://www.ncbi.nlm.nih.gov/pubmed/22393299

3. National Institute of Health and Care Excellence. Acutely ill adults in hospital: recognising and responding to deterioration | Guidance and guidelines | NICE [Internet]. NICE; 2007 [cited 2019 Feb 18]. Available from: https://www.nice.org.uk/guidance/cg50

4. Gao H, McDonnell A, Harrison DA, Moore T, Adam S, Daly K, et al. Systematic review and evaluation of physiological track and trigger warning systems for identifying at-risk patients on the ward. Intensive Care Med [Internet]. 2007 Mar 23 [cited 2019 Feb 18];33(4):667-79. Available from: http://www.ncbi.nlm.nih.gov/pubmed/17318499

5. Morgan RJM, Wright MM. In defence of early warning scores. Br J Anaesth [Internet]. Oxford University Press; 2007 Nov 1 [cited 2019 Feb 18];99(5):747-8. Available from: https://linkinghub.elsevier.com/retrieve/pii/S0007091217347074

6. Morgan R, Williams F, Wright M. An early warning scoring system for detecting developing critical illness. Clin Intensive Care. 1997;(8):100.

7. National Institute for Health and Care Excellence. Acutely ill patients in hospital overview - NICE Pathways [Internet]. 2019 [cited 2019 Feb 18]. Available from: https://pathways.nice.org.uk/pathways/acutely-ill-patients-in-

hospital\#path=view\%3A/pathways/acutely-ill-patients-in-hospital/acutely-ill-patientsin-hospital-overview.xml\&content=view-index

8. Jones M. NEWSDIG: The National Early Warning Score Development and Implementation Group. Clin Med [Internet]. Royal College of Physicians; 2012 Dec [cited 2019 Feb 18];12(6):501-3. Available from: http://www.ncbi.nlm.nih.gov/pubmed/23342400

9. Palla B, Litt IF, Miller KK, Almazan C, Worley M, Soyka LA, et al. Medical complications of eating disorders in adolescents. Pediatrics [Internet]. American Academy of Pediatrics; 1988 May 1 [cited 2019 Feb 18];81(5):613-23. Available from: http://www.ncbi.nlm.nih.gov/pubmed/3162764

10. Misra M, Aggarwal A, Miller KK, Almazan C, Worley M, Soyka LA, et al. Effects of Anorexia Nervosa on Clinical, Hematologic, Biochemical, and Bone Density Parameters in Community-Dwelling Adolescent Girls. Pediatrics. American Academy of Pediatrics; 2004 Dec 1;114(6):1574-83.

11. De Simone G, Scalfi L, Galderisi M, Celentano A, Biase G Di, Tammaro P, et al. Cardiac abnormalities in young women with anorexia nervosa. Br Hear [Internet]. 1994 [cited 2019 Feb 18];71:287-92. Available from: http://heart.bmj.com/

12. Shamim T, Golden NH, Arden M, Filiberto L, Shenker IR. Resolution of vital sign instability: an objective measure of medical stability in anorexia nervosa. J Adolesc Heal [Internet]. Elsevier; 2003 Jan 1 [cited 2019 Feb 18];32(1):73-7. Available from: https://www.sciencedirect.com/science/article/pii/S1054139X02005335

13. McCluskey S, Robinson P. THE MARSI MEWS Dr Sara McCluskey and Dr Paul Robinson. 
Evolution of the MARSI MEWS Results of audit Validation [Internet]. Priory. 2015 [cited 2019 Feb 18]. Available from: https://docplayer.net/53475835-The-marsi-mews-drsara-mccluskey-and-dr-paul-robinson-evolution-of-the-marsi-mews-results-of-auditvalidation.html

14. MARSIPAN TRC of PP and P. MARSIPAN: Management of Really Sick Patients with Anorexia Nervosa 2nd edition CR189 [Internet]. 2014 [cited 2019 Feb 18]. Available from: https://www.rcpsych.ac.uk/docs/default-source/improving-care/better-mhpolicy/college-reports/college-report-cr189.pdf?sfvrsn=6c2e7ada_2

15. Treasure J. A GUIDE TO THE MEDICAL RISK ASSESSMENT FOR EATING DISORDERS [Internet]. King's College London and South London and Maudsley NHS Foundation Trust. 2009 [cited 2019 Feb 18]. Available from: https://www.kcl.ac.uk/ioppn/depts/pm/research/eatingdisorders/resources/GUIDETO MEDICALRISKASSESSMENT.pdf

16. Jones WR, Morgan JF, Arcelus J. Managing physical risk in anorexia nervosa. Adv Psychiatr Treat [Internet]. Cambridge University Press; 2013 May 2 [cited 2019 Feb 18];19(3):201$2 . \quad$ Available from: https://www.cambridge.org/core/product/identifier/S1355514600017363/type/journ al_article

17. QED. Quality network for eating disorders - Standards [Internet]. Royal College of Psychiatrists. 2017. Available from: https://www.rcpsych.ac.uk/improvingcare/ccqi/quality-networks-accreditation/eating-disorders-qed/standards-and-

publications 
Table 1 - Completeness of parameters for the physiological measurements and scoring of the early warning signs system

\begin{tabular}{|l|c|c|c|c|}
\hline Parameter & $\begin{array}{c}\text { R1 Mean } \\
\text { (range) }\end{array}$ & $\begin{array}{c}\text { R1 NA's } \\
\text { (n= 299) }\end{array}$ & $\begin{array}{c}\text { R2 Mean } \\
\text { (Range) }\end{array}$ & $\begin{array}{c}\text { R2 NA scores } \\
\text { (n= 364) }\end{array}$ \\
\hline BMI & $14.1(12.3-18.6)$ & $0[0.0 \%]$ & $14.6(11.8-18.9)$ & $0[0.0 \%]$ \\
\hline RR & $15.94(12-19)$ & $59[19 \%]$ & $15.61(12-19)$ & $3[0.8 \%]$ \\
\hline SO2 & $98.29(95-100)$ & $13[4 \%]$ & $98.2(95-100)$ & $5[1.4 \%]$ \\
\hline T & $36.38(34.9-38)$ & $12[4 \%]$ & $36.51(35.7-37.1)$ & $0[0.0 \%]$ \\
\hline HR & $72.9(46-120)$ & $13[4 \%]$ & $74.88(50-111)$ & $1[0.3 \%]$ \\
\hline Systolic BP & $99.2(56-156)$ & $8[2.7 \%]$ & $101.5(51-144)$ & $0[0.0 \%]$ \\
\hline $\begin{array}{l}\text { Postural drop } \\
\text { score }\end{array}$ & $6.25(0-17)$ & $287[96 \%]$ & $0.44(0-2)$ & $1[0.3 \%]$ \\
Glucose (BM) & 5.56 & $258[86 \%]$ & $6.3(6.3-6.3)$ & $363[99.7 \%]$ \\
\hline AVPU & $\mathrm{A}$ & $7[2.3 \%]$ & $\mathrm{A}$ & $0[0.0 \%]$ \\
\hline MEWS scores & 0.88 & $32[10.7 \%]$ & $0.67(0-6)$ & $4[1.1 \%]$ \\
\hline
\end{tabular}

Legend: Completeness of parameters for the physiological measurements and scoring of the early warning signs system. $\mathbf{B M I}=$ Body mass index; $\mathbf{R R}=$ Respiratory rate; $\mathbf{S 0 2}=$ 0xygen Saturation; HR = Heart rate; $\mathbf{T}=$ Temperature; systolic $\mathbf{B P}=$ systolic blood pressure; Postural $\mathbf{B P}=$ Blood pressure postural drop (sitting minus standing $\mathrm{BP}$ ); $\mathbf{B M}=$ blood glucose measurement; AVPU = Alertness score 


\section{Figure 1 - Comparative results of completeness from R1 and R2 of the audit cycle}

Proportion of physical variables correctly recorded during MARSI MEWS scoring

(\%)

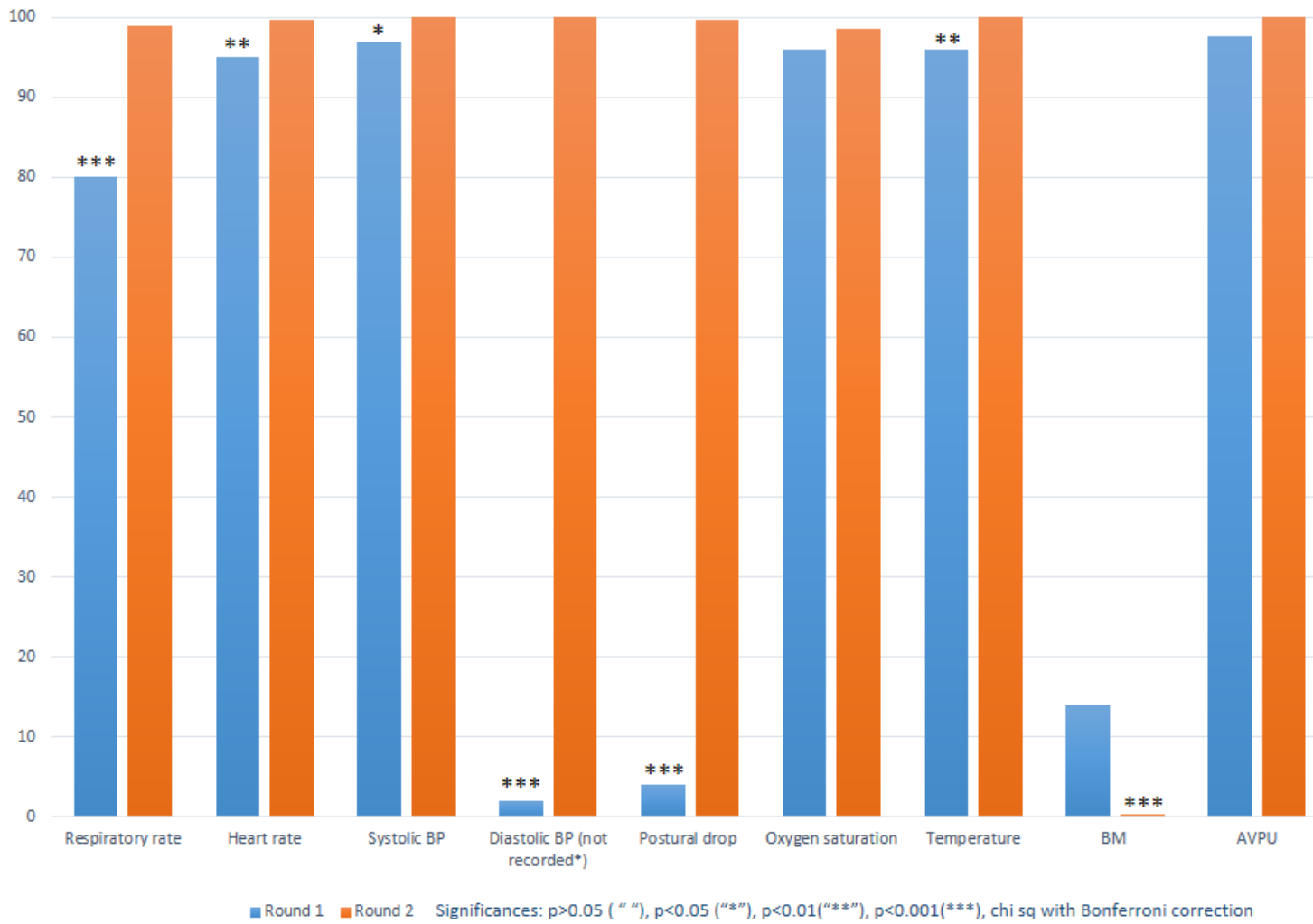

Legend - Physiological parameters measured on the inpatient setting for the calculation of the early warning signs score; colour coding for different rounds of the audit: Round 1 (blue), Round 2 (orange); $\mathbf{R R}=$ Respiratory rate; $\mathbf{S 0 2}=$ 0xygen Saturation; HR = Heart rate; $\mathbf{T}=$ Temperature; systolic BP = systolic blood pressure; Postural BP = Blood pressure postural drop; $\mathbf{B M}=$ blood glucose measurement; AVPU = Alertness score; 


\section{FIGURE 2 - Sepsis indicator summary results}

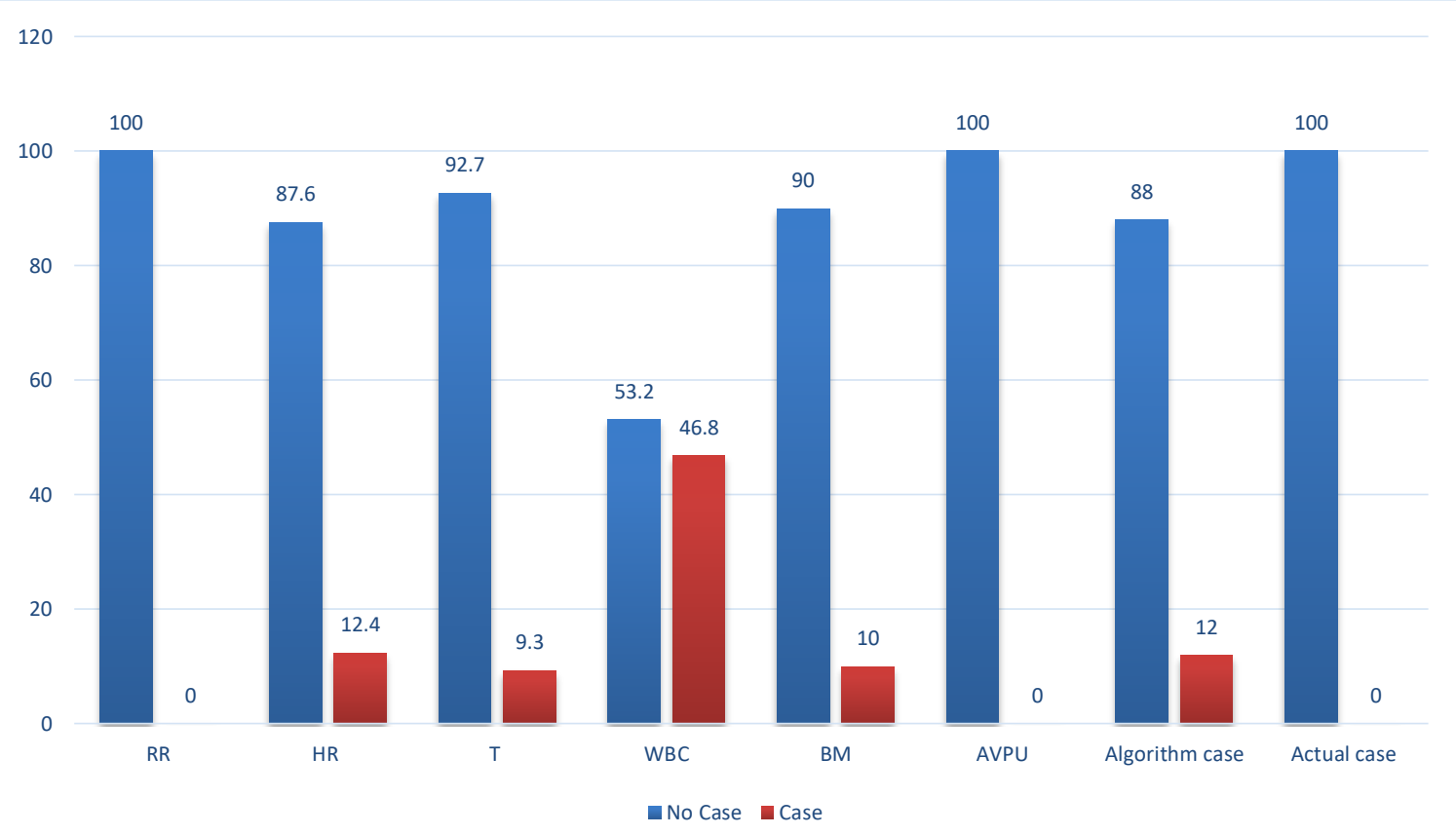

Legend: Sepsis indicator summary results from Round 1; the percentages of sepsis cases identified by the sepsis indicator are presented in red. There were no actual sepsis cases in Round 1 or 2 . Round 2 identified no sepsis cases (actual or algorithmic). 